\title{
SOME CHARACTERIZATIONS OF $\pi$-REGULAR RINGS WITH NO INFINITE TRIVIAL SUBRING
}

\author{
by YASUYUKI HIRANO
}

(Received 4th September 1988)

Dedicated to Professor Hiroyuki Tachikawa on his 60th birthday

\begin{abstract}
It is shown that a ring $R$ is a $\pi$-regular ring with no infinite trivial subring if and only if $R$ is a subdirect sum of a strongly regular ring and a finite ring. Some other characterizations of such a ring are given. Similar result is proved for a periodic ring. As a corollary, it is shown that every $\delta$-ring is a subdirect sum of a finite ring and a commutative ring. This was conjectured by Putcha and Yaqub.
\end{abstract}

1980 Mathematics subject classification (1985 Revision): 16A30.

In [7] Laffey proved that if $R$ is an infinite periodic ring which is orthogonally finite and with all trivial subrings finite, then $R$ has a commutative ideal $I$ such that $R / I$ is finite. Recently Armendariz [1] showed that such a ring is described as follows: $R=F \oplus S$, where $F$ is a finite ring and $S$ is a finite direct sum of periodic fields. He also gave an analogue result for strongly $\pi$-regular rings. More recently, in [6] we removed the assumption of orthogonal finiteness, and proved that a $\pi$-regular ring (resp. periodic ring) $R$ with no infinite trivial subring has a strongly regular ideal (resp. commutative ideal) $M$ such that $R / M$ is finite. In this paper, we will show that a ring $R$ is a $\pi$-regular ring (resp. periodic ring) with no infinite trivial subring if and only if $R$ is a subdirect sum of a strongly regular ring (resp. $J$-ring) and a finite ring. We will also provide several characterizations of $\pi$-regular rings (resp. periodic rings) with no infinite trivial subring. As a result, we will solve the open problem of Putcha and Yaqub in [8] affirmatively.

The Jacobson radical of a ring $R$ will be denoted by $J(R)$, and the prime radical by $P(R)$. The ring of integers will be denoted by $\mathbf{Z}$.

A ring $R$ is called $\pi$-regular if for each $x$ in $R$ there exists a positive integer $n$ (depending on $x$ ) and an element $y$ of $R$ such that $x^{n}=x^{n} y x^{n}$. A $\pi$-regular ring $R$ for which the $n$ in the above can be taken to be 1 for all $x$ is called von Neumann regular. A strongly regular ring is a von Neumann regular ring with no nonzero nilpotent element. By a trivial subring of a ring $R$ we mean a subring $S$ of $R$ with $S^{2}=0$.

Theorem 1. The following conditions are equivalent for a ring $R$.

(1) $R$ is a $\pi$-regular ring with no infinite trivial subring. 
(2) $R$ has a strongly regular ideal $M$ such that $R / M$ is a finite ring.

(3) $R$ is a subdirect sum of a strongly regular ring and a finite ring.

(4) $R$ has a finite ideal $I$ such that $R / I$ is strongly regular.

(5) $P(R)$ is finite and $R / P(R)$ is the direct sum of a strongly regular ring and a finite ring.

(6) $R$ is a $\pi$-regular ring with only finitely many nilpotent elements.

(7) There exists a finite subset $S$ of $R$ such that for each $x \in R$, there is $a \in R$ such that $a x=x a$ and $x-x^{2} a \in S$.

Proof. $(1) \Rightarrow(2)$. This was proved in [6].

(2) $\Rightarrow(3)$. Take an ideal $I$ of $R$ which is maximal with respect to the property that I $\cap M=0$. We claim that $R / I$ has no nonzero nilpotent element. Let $a$ be an element of $R$ such that $a^{2} \in I$ and let $m \in M$. Then we see $(a m a)^{2} \in M \cap I=0$. Since $a m a \in M$ and since $M$ has no nonzero nilpotent element, we get $a m a=0$. Then we obtain $m a=0$, because $m a \in M$ and $(m a)^{2}=0$. Thus we get $M a=0$. Similarly we obtain $a M=0$. Now let $A=M \cap(R a+a R+Z a+I)$. Then we see that $A^{2} \subset M(R a+a R+Z a+I) \subset M \cap I=0$. Since $A \subset M$, we get $A=0$. By the choice of $I$, we conclude that $a \in I$. This proves that $R / I$ has no nonzero nilpotent element. Let $b$ be an arbitrary element of $R$. Since $R / M$ is a finite ring, it is $\pi$-regular, and so there exists a positive integer $n$ and $x \in R$ such that $b^{n}-b^{n} x b^{n} \in M$. Since $M$ is strongly regular, there exists $y \in M$ such that $b^{n}-b^{n} x b^{n}=$ $\left(b^{n}-b^{n} x b^{n}\right) y\left(b^{n}-b^{n} x b^{n}\right)$. Then it holds that $b^{n}=b^{n} z b^{n}$, where $z=x+y-x b^{n} y-y b^{n} x+$ $x b^{n} y b^{n} x$. Therefore $R$, and hence $R / I$, is $\pi$-regular. As shown above, $R / I$ has no nonzero nilpotent element, and hence $R / I$ is strongly regular. Thus $R$ is a subdirect sum of the strongly regular ring $R / I$ and the finite ring $R / M$.

(3) $\Rightarrow(4)$. By hypothesis, there exist ideals $I$ and $M$ with $I \cap M=0$ such that $R / I$ is strongly regular and $R / M$ is finite. Then $I$ can be embedded in the finite ring $R / M$, and so $I$ is finite.

$(4) \Rightarrow(5)$. Since every element of $P(R)$ is nilpotent and since $R / I$ has no nonzero nilpotent element, we get $P(R) \subset I$. Hence $P(R)$ is finite. Let $R^{\prime}=R / P(R)$, and $I^{\prime}=I / P(R)$. We claim that $I^{\prime}$ is a semisimple ring. Suppose, to the contrary, that $J\left(I^{\prime}\right) \neq 0$. Since $I^{\prime}$ is a finite ring, $J\left(I^{\prime}\right)$ is nilpotent. Hence $I^{\prime} J\left(I^{\prime}\right)$ is a nilpotent left ideal of $R^{\prime}$. Since $P\left(R^{\prime}\right)=0$, we get $I^{\prime} J\left(I^{\prime}\right)=0$. Thus $\left(R^{\prime} J\left(I^{\prime}\right)\right)^{2}=R^{\prime} J\left(I^{\prime}\right) R^{\prime} J\left(I^{\prime}\right) \subset I^{\prime} J\left(I^{\prime}\right)=0$, and hence $J\left(I^{\prime}\right)=0$. Thus the finite semisimple ring $I^{\prime}$ has an identity $e$ (see e.g. [5, Corollary 2, p. 30]). Since $I^{\prime}$ is an ideal of $R^{\prime}$, we can easily see that $e$ is a central idempotent of $R^{\prime}$. Hence we have the decomposition $R^{\prime}=I^{\prime} \oplus S$, where $S=\left\{r-r e \mid r \in R^{\prime}\right\}$. Since $S$ is isomorphic to $R / I, S$ is strongly regular.

$(5) \Rightarrow(6)$. By hypothesis $R$ has a finite ideal $I(\supset P(R))$ such that $R / I$ is strongly regular. Since $R / I$ has no nilpotent element, all nilpotent elements of $R$ are contained in the finite ideal $I$. Hence those forms a finite set. To prove the $\pi$-regularity of $R$, take an arbitrary prime ideal of $R$. Then, by hypothesis, $R / P$ is either a division ring or a finite simple ring. Obviously $R$ is of bounded index. Therefore, by Corollary 2.2 of [4], we conclude that $R$ is $\pi$-regular. 
$(6) \Rightarrow(7)$. Since $R$ is of bounded index, $R$ is strongly $\pi$-regular by Theorem 5 of [2]. Let $S$ be the set of all nilpotent elements of $R$ and let $x$ be any element of $R$. By Theorem 3 and Lemma 4 of [2] there exists $a \in R$ such that $x a=a x$ and $x-x^{2} a \in S$.

$(7) \Rightarrow(1)$. It is easy to see that all trivial subrings of $R$ are contained in $S$. Hence $R$ has no infinite trivial subring. To prove the $\pi$-regularity of $R$, take an arbitrary element $x$ of $R$. By hypothesis there exists $a_{1} \in R$ such that $a_{1} x=x a_{1}$ and $x-x^{2} a_{1} \in S$. Let us set $b_{1}=x-x^{2} a_{1}$. Inductively, if $b_{n-1} \in S$ is defined, then we obtain $a_{n} \in R$ such that $a_{n} b_{n-1}^{2}=b_{n-1}^{2} a_{n}$ and $b_{n-1}^{2}-b_{n-1}^{4} a_{n} \in S$, and then we define $b_{n}=b_{n-1}^{2}-b_{n-1}^{4} a_{n}$. Since $S$ is finite, there are positive integers $k<m$ such that $b_{k}=b_{m}$. Then we get $y, z \in R$ such that $x^{2^{k-1}}=x^{2^{k-1}+1} y=z x^{2^{k-1}+1}$. If we set $n=s^{k-1}, a=y^{n}$, and $b=z^{n}$, then we have $x^{n}=x^{2 n} a=b x^{2 n}$. Hence we obtain $x^{2 n}=x^{2 n} a b x^{2 n}$. This proves that $R$ is $\pi$-regular.

Since the condition (7) of Theorem 1 is inherited by every homomorphic image of $R$, we have the following:

Corollary 1. If $R$ is a $\pi$-regular ring with no infinite trivial subring, then so is every homomorphic image of $R$.

The following example shows that, in the condition (3) of Theorem 1, "subdirect sum" can not be replaced by "direct sum",

Example. Let $K$ be a finite field, $I$ an infinite set, and let $K^{I}$ denote the direct product of the $I$-copies of $K$. Take a maximal ideal $M$ of $K^{I}$ containing the direct sum $K^{(I)}$ of the $I$-copies of $K$. Let $n$ be the order of $K$. Then the field $K^{I} / M$ satisfies the identity $X^{n}-X=0$. Hence we conclude that $K^{I} / M$ is isomorphic to the field $K$. Let $R$ be the additive abelian group $K^{I} \oplus\left(K^{I} / M\right)$ and define multiplication in $R$ by $(a, r)$ $(b, s)=(a b, a s+r b)$. Then $R$ is a ring satisfying the equivalent conditions of Theorem 1 . In fact, $\left(K^{I} / M\right)^{*}=\left\{(0, r) \mid r \in K^{I} / M\right\}$ is a finite ideal of $R$, and $R /\left(K^{I} / M\right)^{*}\left(\simeq K^{I}\right)$ is strongly regular, and hence $R$ satisfies the condition (4) of Theorem 1 . It is easy to see that $R$ cannot be represented as the direct sum of a strongly regular ring and a finite ring.

A ring $R$ is said to be periodic if for each $x \in R$, there exist distinct positive integers $m$, $n$ for which $x^{m}=x^{n}$. A ring $R$ is called a $J$-ring if for each $x \in R$, there exists an integer $n>1$ such that $x=x^{n}$. We can easily see that a ring $R$ is a $J$-ring if and only if $R$ is a periodic ring with no nonzero nilpotent element. Finally, following [8], a ring $R$ is called a $\delta$-ring if $R$ has a finite subset $S$ such that for each $x \in R$, there exists a polynomial $f(X) \in \mathbf{Z}[X]$ such that $x-x^{2} f(x) \in S$.

Theorem 2. The following conditions are equivalent for a ring $R$.

(1) $R$ is a periodic ring with no infinite trivial subring.

(2) $R$ has a $J$-ideal $M$ such that $R / M$ is a finite ring.

(3) $R$ is a subdirect sum of $a J$-ring and a finite ring.

(4) $R$ has a finite ideal $I$ such that $R / I$ is a $J$-ring. 
(5) $P(R)$ is finite and $R / P(R)$ is the direct sum of a strongly regular ring and a finite semisimple ring.

(6) $R$ is a periodic ring with only finitely many nilpotent elements.

(7) $R$ is $a$-ring.

In preparation for the proof of Theorem 2, we state two lemmas the first of which is Proposition 2 of [3].

Lemma 1 ([3]). Let $R$ be a ring. Suppose that for each $x \in R$, there exists a positive integer $n$ and a polynomial $p(X) \in \mathbf{Z}[X]$ for which $x^{n}=x^{n+1} p(x)$. Then $R$ is periodic.

Lemma 2. Let $I$ be an ideal of a ring $R$. Then $R$ is periodic if and only if both $I$ and $R / I$ are periodic.

Proof. It suffices to prove the "if" part. Let $x$ be an arbitrary element of $R$. Since $R / I$ is periodic, there exist positive integers $n<m$ such that $x^{n}-x^{m} \in I$. Since $I$ is periodic, there exist positive integers $k<h$ such that $\left(x^{n}-x^{m}\right)^{k}=\left(x^{n}-x^{m}\right)^{h}$. This equation can be rewritten in the form $x^{n k}=x^{n k+1} p(x)$ for some $p(X) \in \mathbf{Z}[X]$. Hence, by Lemma $1, R$ is periodic. 1.

Now we can prove Theorem 2. The proof proceeds parallel to the proof of Theorem

Proof of Theorem 2. (1) $\Rightarrow(2)$. This was shown in the proof of Corollary 1 of $[6$.

$(2) \Rightarrow(3)$. As shown in the proof of $(2) \Rightarrow(3)$ of Theorem 1 , there exists an ideal $I$ of $R$ with $I \cap M=0$ such that $R / I$ has no nonzero nilpotent element. By virtue of Lemma 2 , we can easily see that $R / I$ is a $J$-ring. Therefore $R$ is a subdirect sum of the $J$-ring $R / I$ and the finite ring $R / M$.

$(3) \Rightarrow(4)$ and $(4) \Rightarrow(5)$ can be shown by the similar way as in the corresponding parts of the proof of Theorem 1 .

$(5) \Rightarrow(6)$. Using Lemma 2 , we can prove that $R$ is periodic. By the same argument as in the proof of $(5) \Rightarrow(6)$ of Theorem 1 , we also see that $R$ has only finitely many nilpotent elements.

$(6) \Rightarrow(7)$. Let $N$ be the set of all nilpotent elements of $R$ and let $x$ be an arbitrary element of $R$. Since $R$ is periodic, there exist positive integers $m$ and $n$ such that $x^{m}=x^{m+n}$. Then we have that $x-x^{n+1} \in N$. By hypothesis, $N$ is finite, and hence $R$ is a $\delta$-ring.

$(7) \Rightarrow(1)$. It was proved in Lemma 2 of [8] that $R$ satisfies the hypothesis of Lemma 1 . Hence $R$ is periodic. Since every subring of a $\delta$-ring is a $\delta$-ring, $R$ has no infinite trivial subring by Theorem 1 of [8].

The following corollary is the answer to the question of Putcha and Yaqub [8, p. 20].

Corollary 2. Every $\delta$-ring is a subdirect sum of a finite ring and a commutative ring. 
Proof. A well known theorem of Jacobson says that every $J$-ring is commutative (see [5, Theorem 3.1.2]). Hence our assertion follows from the equivalence of the conditions (3) and (7) of Theorem 2.

\section{REFERENCES}

1. E. P. Armendariz, On infinite periodic rings, Math. Scand. 59 (1986), 5-8.

2. G. Azumaya, Strongly $\pi$-regular rings, J. Fac. Sci. Hokkaido Univ. 13 (1954), 34-39.

3. M. Chacron, On a theorem of Herstein, Canad. J. Math. 21 (1969), 1348-1353.

4. J. W. Fisher and R. L. SNider, On the von Neumann regularity of rings with regular prime factor rings, Pacific J. Math. 54 (1974), 135-144.

5. I. N. Herstein, Noncommutative Rings (Carus Monogr. No. 15, Amer. Math. Ass., New York, 1968).

6. Y. Hirano, On $\pi$-regular rings with no infinite trivial subring, Math. Scand., to appear.

7. T. J. LAFFEY, Commutative subrings of periodic rings, Math. Scand. 39 (1976), 161-166.

8. M. S. Putcha and A. YaQub, A finiteness conditions for rings, Math. Japon. 22 (1977), 13-20.

Department of Mathematics

Okayama University

Orayama 700

JAPAN 